Gabriel, Thomas; Kühne, Elke; Faulhaber, Petra; Promny, Markus; Horchler, Peter Sohlenstabilisierung und Erosionseindämmung am Beispiel der Elbe

Verfügbar unter / Available at:

https://hdl.handle.net/20.500.11970/100654

Vorgeschlagene Zitierweise / Suggested citation:

Gabriel, Thomas; Kühne, Elke; Faulhaber, Petra; Promny, Markus; Horchler, Peter (2011):

Sohlenstabilisierung und Erosionseindämmung am Beispiel der Elbe. In: Wasserwirtschaft Jg. 101 (6/2011). S. 27-32. 
Erstveröffentlichung in WasserWirtschaft 6 (2011), S. 27-32.

Für eine korrekte Zitierbarkeit ist die Seitennummerierung der Originalveröffentlichung für jede Seite kenntlich gemacht.

\section{Sohlenstabilisierung und Erosionseindämmung am Beispiel der Elbe}

Thomas Gabriel, Elke Kühne, Petra Faulhaber, Markus Promny und Peter Horchler

An einem Abschnitt der MittelElbe hat sich die Gewässersohle über einen Zeitraum von mehr als 100 Jahren um durchschnittlich einen Meter eingetieft. Diese Erosionstendenzen stellen eine zunehmende Gefährdung für die Funktionsfähigkeit des Stromregelungssystems dar. Auch sind zukünftige Auswirkungen auf die Umwelt nicht auszuschließen. Auf der Grundlage eines Sohlenstabilisierungskonzeptes werden von der Wasser- und Schifffahrtsverwaltung des Bundes notwendige Maßnahmen zur Eindämmung der Erosion bei gleichzeitiger BElbehaltung der natürlichen Sohlendynamik umgesetzt.

\section{Die Erosionsstrecke der Elbe}

Als Erosion wird eine langfristige und großräumige Eintiefung der Flusssohle bezeichnet. Seit mehr als 100 Jahren lässt sich in einem Abschnitt der oberen Mittelelbe Sohlenerosion beobachten, weshalb dieser Abschnitt "Erosionsstrecke" genannt wird. Wurde zunächst der Bereich zwischen Mühlberg (Elbe-km 120) und Coswig (Elbe-km 230) so bezeichnet, wird heute auch die sich unterstromig anschließende Strecke bis zur Saalemündung (Elbe-km 290,7) mit einbezogen (Bild 1). Die Erosion der Sohle geht mit einem entsprechenden Absinken des Flusswasserspiegels einher (Bild 2). Sie hat u. a. nachteilige Auswirkungen auf die Schifffahrtsverhältnisse und auch auf die Bauwerkssicherheit des bestehenden Stromregelungssystems. Da auch das flussnahe Grundwasser vom Wasserspiegelabsunk betroffen ist, sind 


\section{Autorenfassung}

Gabriel, Kühne, Faul haber, Promny, Horchler: Sohlenstabilisierung und Erosionseindämmung am Beispiel der Elbe, 2011

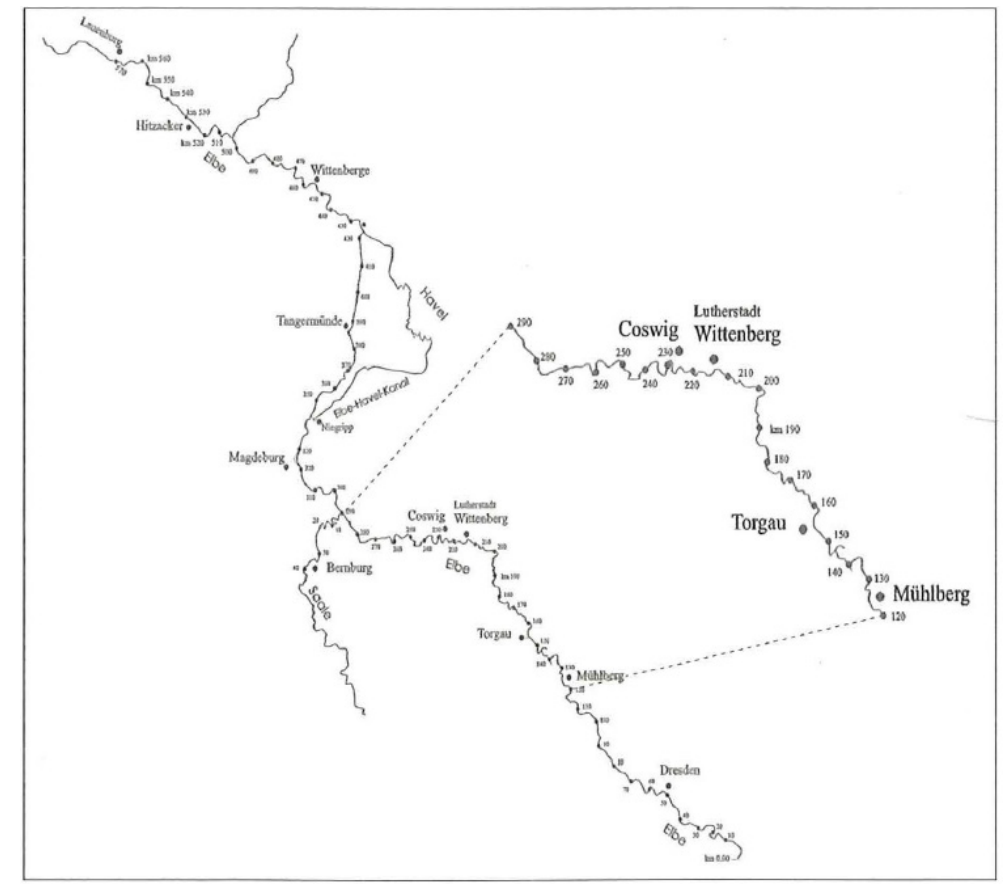

Bild 1: Die Erosionsstrecke der Elbe

Beeinträchtigungen für das Ökosystem der Talaue nicht auszuschließen.

Um einer weiter fortschreitenden Erosion entgegen zu wirken und die Sohle zu stützen, wird der Elbe seit Mitte der 1990er Jahre Geschiebeersatzmaterial in wechselnden Abschnitten der Erosionsstrecke zugeführt [1]. Für eine nachhaltige Eindämmung der Erosion sind jedoch flankierende Maßnahmen im Bereich des Gewässerbettes und in den Vorländern notwendig. Hierfür hat die Wasser- und Schifffahrtsverwaltung des Bundes (WSV) im Jahr 2009 in Zusammenarbeit mit den Bundesanstalten für Wasserbau (BAW) und Gewässerkunde (BfG) sowie mit Unterstützung der Bundesländer Sachsen und Sachsen-Anhalt das "Sohlenstabilisierungskonzept für die Elbe von Mühtberg bis zur Saalemündung" [2] aufgestellt. Es ist seitdem Planungs-und Handlungsgrundlage für die Unterhaltungsmaßnahmen der WSV in diesem Elbabschnitt.

Die Elbe unterliegt im hier betrachteten Stromabschnitt keinerStauregelung und hat freien Abfluss. Sie ist ein anthropogen überprägter Fluss. Typische künstliche Strukturelemente sind neben den Hochwasserdeichen vor allem die Buhnen und Deckwerke, die das Flussbett seit der Mittelwasserregelung in den 80er Jahren des 19. Jahrhunderts im Lauf fixieren.

Kennzeichnend für die Erosionsstrecke der Elbe ist ein defizitärer Sedimenthaushalt, der einen räumlich ausgedehnten Trend zur Eintiefung der Gewässersohle zur Folge hat. Diese Entwicklung ist in der sedimentologisch-morphologischen Charakteristik der Erosionsstrecke begründet, 
Gabriel, Kühne, Faulhaber, Promny, Horchler: Sohlenstabilisierung und Erosionseindämmung am Beispiel der Elbe. WasserWirtschaft 6 (2011), S. 27-32.

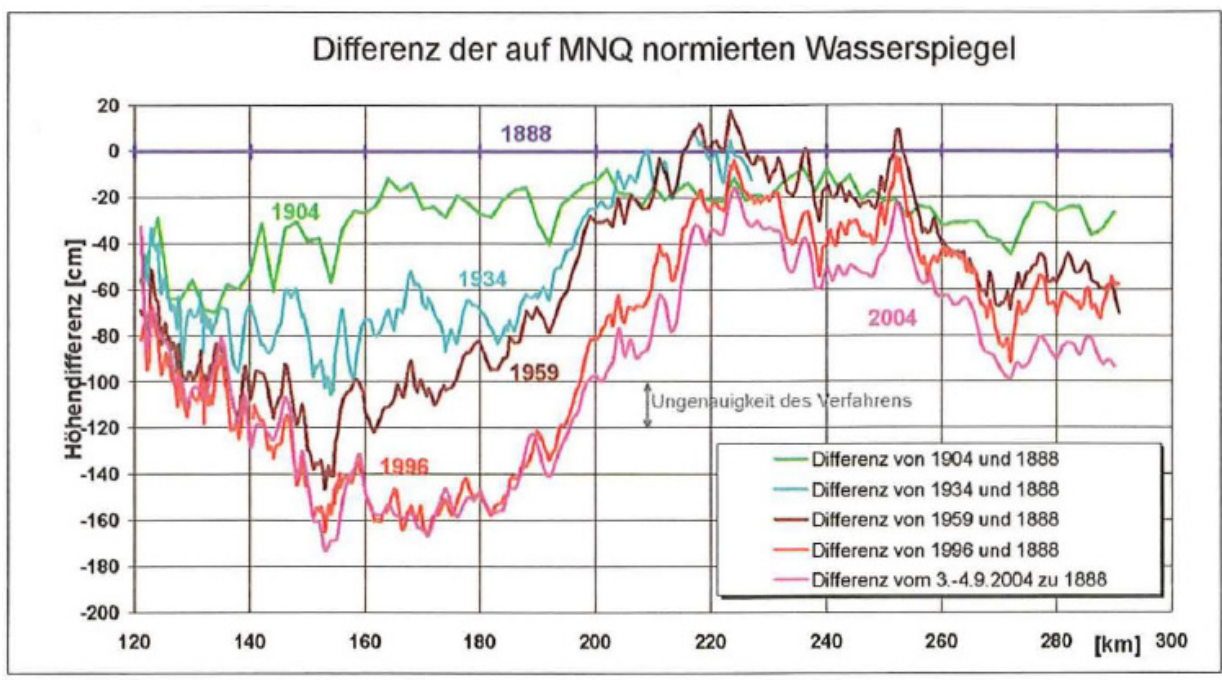

Bild 2: $\quad$ Wasserspiegelentwicklung in Bezug auf einen normierten Wasserspiegel von 1888 (BAW)

wird aber auch stark durch anthropogene Eingriffe aus der Vergangenheit beeinflusst. Zu nennen sind hier u. a. ein zu geringer Geschiebeeintragals Folge des Baus von Staustufen und Querbauwerken im Oberlauf und in den einmündenden Nebengewässern sowie die Verminderung der Geschiebeeinträge durch Seitenerosion, nachdem die Hoch- und Mittelwasserbereiche durch bauliche Maßnahmen festgelegt wurden.

Aus der historischen Analyse der Wasserstände lässt sich eine verstärkte Erosion bereits Ende des 19. Jahrhunderts nachweisen. Die Erosion hat mit großer Wahrscheinlichkeit auch schon früher eingesetzt. Die Vergleiche von Messungen des Wasserspiegels, geometrischen Sohlenaufnahmen und Feststofftransportmessungen zeigen, dass der Eintiefungsprozess weiter anhält. Nach aktuellen Analysen und Modelluntersuchungen wandert der Schwerpunkt der Erosion langsam stromabwärts (Bild 2).

Lag in der Vergangenheit das Erosionsmaximum im Bereich des T01·gauer Felsens (Elbe-km 155), wo die Erosion aktuell zum Abklingen kommt, so trat zwischen den 1960er und 1980er Jahren die stärkste Erosion bei Pretzsch (Elbe-km 185) auf. In den letzten 15 bis 20 Jahren zeichnet sich eine weitere Verlagerung des Erosionsschwerpunktes über Klöden (Elbe-km 190) und Wittenberg/L. (Elbe-km 215) in Richtung Saalemündung (Elbe-km 291) ab. Die Eintiefung erfolgt dabei nicht kontinuierlich und nicht gleichmäßig über die gesamte Strecke. Trotz langfristiger und großräumiger 


\section{Autorenfassung}

Gabriel, Kühne, Faul haber, Promny, Horchler: Sohlenstabilisierung und Erosionseindämmung am Beispiel der Elbe, 2011

Erosion kommt es u. a. auch aufgrund eines nicht voll funktionsfähigen Stromregelungssystems mit einer ungleichmäßiger Streichlinienführung lokal und temporär zu Anlandungen, die zu Fehlstellen für die Schifffahrt werden.

Die anhaltendeErosion hat bereichsweise dazu geführt, dass die Regelungsbauwerke im Hinblick auf den Bezugswasserstand zu hoch liegen und somit auch über Mittelwasser hinaus verstärkt regelungswirksam sind. Gleichzeitig hat sich der Abstand zwischen dem Niveau der Vorländer und der Flusssohle vergrößert. Als Folge davon werden die Vorländer erst bei höheren Abflüssen und somit später sowie seltener überströmt. Diese Wirkungen führen zu einer Selbstverstärkung der Erosion und bestätigen somit gleichzeitig die dringende Handlungsnotwendigkeit, diesen Tendenzen entgegenzuwirken.

\section{Das Sohlenstabilisierungskonzept}

Grundlage für die Entwicklung des Konzeptes zur Eindämmung der Erosionstendenzen war ein umfangreiches Untersuchungsprogramm, welches maßnahmenbegleitend fortgeführt wird. Zwar konnte auf Erfahrungen aus der Vergangenheit und aus anderen Flussgebieten mit vergleichbarer Problematik zurückgegriffen werden, aber eigene aktuelle gebietsspezifische Untersuchungen in der Natur [3) sowie mit Modellen ([4), [5]) erwiesen sich als unverzichtbar, um die ablaufenden Naturprozesse hinreichend genau beschrElben zu können.

\section{Ziele und Grundsätze}

Als Entwicklungsziel für die Erosionsstrecke wird die Stabilisierung der mittleren Sohlenhöhe bei Erhalt oder Förderung der morphologischen Dynamik angestrebt.

Neben der Aufrechterhaltung bzw. der Wiederherstellung der definierten Schifffahrtsbedingungen und der Beachtung der Hochwasserneutralität bei den zu realisierenden Maßnahmen sind aus ökologischer Sicht eine stärkere Strukturierung und Dynamisierung des Flusslaufes und der Aue insbesondere durch Zulassen von Struktur bildenden Prozessen sowie eine häufigere und großräumigere Überströmung der Aue bzw. der Vorländer zu berücksichtigen.

Die konkreten Maßnahmen zur Erosionsminderung werden u. a. unter Berücksichtigung der nachfolgenden Grundsätze gewählt:

- Dem Geschiebedefizit wird durch eine dauerhafte, über die gesamte Erosionstrecke verteilte Geschiebezugabe begegnet.

- Die Regelungsbauwerke und das Gewässerbett einschließlich der bei häufigem Hochwasser $\left(<\mathrm{HQ}_{5}\right)$ überströmten Vorländer sind so zu gestalten, dass der gleichmäßige Durchtransport von Geschiebe gewährleistet wird.

- Ungleichmäßigkeiten im Frachtverlauf, die sich durch Regelungsmaßnahmen nicht beheben lassen, werden durch gezielte Geschiebeumlagerungen ausgeglichen. 


\section{Autorenfassung}

Gabriel, Kühne, Faul haber, Promny, Horchler: Sohlenstabilisierung und Erosionseindämmung am Beispiel der Elbe, 2011

- Erosionsbedingt beeinträchtigte Strukturen im Vorland, wie Flutrinnen und Mäanderwege (Altarme), werden durch Impulsmaßnahmen reaktiviert.

\section{Maßnahmen}

Die Untersuchungen zeigten, dass der Geschiebezugabe bzw. der Geschiebeumlagerung weiterhin eine zentrale Rolle zufallen wird. Darüberhinaus wird ein wesentlicher Schwerpunkt auf der Umgestaltung der Regelungsbauwerke liegen. U.a. bedeutet das, die Buhnen in ihrer Höhe - anzupassen. Auch sollen in ihrer hydraulischen und ökologischen Wirkungsweise optimierte Bauformen angewendet werden. Regelungstechnisch nicht mehr erforderliche Bauwerke werden zurückgebaut.

Durch Uferabgrabungen und den Abtrag von Uferrehnen soll dem Fluss ein früheres Ausufern ermöglicht werden. Durch das Reaktivieren von Flutrinnen und das Wiederanbinden von Altarmen wird das Gewässerbett ebenfalls entlastet und der Strömungsangriff auf die Sohle bei Abflüssen über Mittelwasser reduziert.

Die laufende Unterhaltung wird mit Blick auf die Erosionsprobleme optimiert.

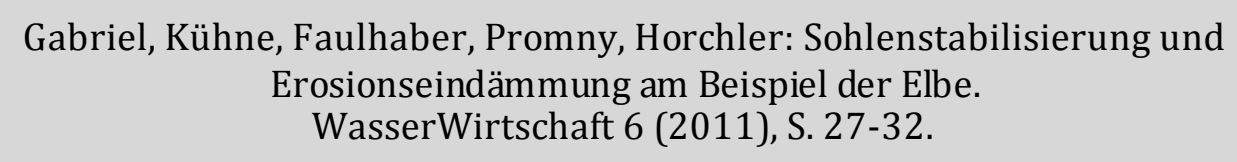

Beispielsweise wird Sediment aus verlandeten Buhnenfeldern entnommen. Dadurch vergrößert sich die Wasserfläche bei niedrigen Abflüssen und die Wassertiefe in den Buhnenfeldern bei allen Abflüssen. Die hydraulische Wirksamkeit der umströmten Buhnen wird wieder hergestellt.

Aufgrund der großen Ausdehnung der Erosionsstrecke und der Notwendigkeit, sich bei der Umsetzung von Schwerpunktmaßnahmen zuerst auf die Bereiche mit den größten Erosionstendenzen zu konzentrieren, wird für die Erosionsstrecke der Elbe eine Unterteilung in Streckenabschnitte von 15 bis 30 km Länge gleicher oder annähernd gleicher Charakteristik vorgenommen (Tabelle 1).

\section{Naturschutzfachliche Bewertung der Maßnahmen gegen die Erosion}

Die Erosionsstrecke der Elbe befindet sich in einem ökologisch bedeutsamen Gebiet und die Maßnahmen, die hier umgesetzt werden, haben auch Auswirkungen auf die hiervon betroffenen Lebensräume. Obwohl die Eindämmung der Erosionstendenzen grundsätzlich auch aus Sicht des Naturschutzes zu begrüßen ist, wurden die im Sohlenstabilisierungskonzept entwickelten Maßnahmen hinsichtlich ihrer ökologischen Wirkungen betrachtet und einer naturschutzfachlichen Bewertung unterzogen. 


\section{Autorenfassung}

Gabriel, Kühne, Faul haber, Promny, Horchler: Sohlenstabilisierung und Erosionseindämmung am Beispiel der Elbe, 2011

Als Fazit bleibt dabei festzuhalten, dass die Eindämmung der fortschreitenden Erosion eine Voraussetzung für den Erhalt der auentypischen Funktionen darstelltund die Maßnahmen im Vorland gut geeignet sind, die naturschutzfachlichen Ziele einer Redynamisierung des Auenbereichs und dem Anstoßen auentypischer Prozesse auf größeren Flächeneinheiten zu ermöglichen sowie generell die Entwicklungsziele nationaler und internationaler Schutzgebiete zu unterstützen.

\section{Pilotmaßnahme Klöden}

Die Strecke Klöden umfasst den Abschnitt zwischen Prettin bis zur Mündung der Elster (Elbe-km 170 bis 198,5). Hier hat sich die mittlere Stromsohle seit Ende des 19. Jahrhunderts um ca. 1,6 m eingetieft [6]. Die Auswirkungen der lang anhaltenden Erosion verdeutlichen in diesem Abschnitt der Elbe die besondere Dringlichkeit für die Umsetzung von Maßnahmen. Als weiterer Grund für Klöden als Pilotstrecke sind die großen ökologischen Entwicklungspotenziale im Bereich des Gewässerbettes sowie in den Vorlandbereichen zu nennen. Die Maßnahmen konzentrieren sich in der Strecke Klöden auf den Bereich Elbe-km 185,5 bis 196,6 (Bild 3).

Schon in der Vergangenheit wurde die Sohle an Problemstellen (z. B. Klödener Bogen) lokal durch Sohlenahdeckungen stabilisiert. Ergänzend wird das Geschiebedefizit seit 1996 durch Einbringen von Geschiebeersatzmaterial vermindert [1]. Das vom Sollzustand abweichende Regelungssystem wurde durch diese Maßnahmen jedoch nicht verändert, so dass Unregelmäßigkeitenim Geschiebetransport entlang der Fließstrecke, die zu starke Regelung für Abflüsse über Mittelwasser sowie die mangelnde Gewässervernetzungweiterhin Bestand haben. Hier muss durch bauliche Maßnahmen Abhilfe geschaffen werden.

Voraussetzung für die Ermittlung der jeweils geeigneten Maßnahmenkombination

\begin{tabular}{|l|l|l|l|}
\hline Nr. & Streckenbezeichnung & Streckenabschnitt & $\begin{array}{l}\text { Schwerpunktmaßnahmen zur } \\
\text { Sohlstabilisierung neben der } \\
\text { Regelunterhaltung }\end{array}$ \\
\hline 1 & Mühlberg & Kreinitz bis Belgern & $\begin{array}{l}\bullet \text { Geschiebezugabe } \\
\bullet \text { Geschiebeumlagerung } \\
\text { Grobkornanreicherung }\end{array}$ \\
\hline 2 & Torgau & Belgern bis Prettin & $\begin{array}{l}\bullet \text { Geschiebezugabe } \\
\text { Grobkornanreicherung } \\
\text { Modifikation der Rege- } \\
\text { lungsbauwerke } \\
\text { Vergrößerung des Abfluss- } \\
\text { anteils der Vorländer }\end{array}$ \\
\hline 3 & Klöden & Elbe-km 140 bis Elbe-km 170) & \begin{tabular}{l} 
Geschiebezugabe \\
\hline
\end{tabular} \\
\hline
\end{tabular}




\section{Autorenfassung}

Gabriel, Kühne, Faul haber, Promny, Horchler: Sohlenstabilisierung und Erosionseindämmung am Beispiel der Elbe, 2011

\begin{tabular}{|c|c|c|c|}
\hline & & (Elbe-km 170 bis Elbe-km 198,5) & $\begin{array}{ll}\text { - } & \text { Modifikation der Rege- } \\
\text { lungsbauwerke } \\
\text { - } \\
\text { - } \\
\text { Uferabgrabung } \\
\text { anteils der Vorländer }\end{array}$ \\
\hline 4 & Wittenberg/ L. & $\begin{array}{l}\text { Elster-Mündung bis Coswig/Anh. } \\
\text { (Elbe-km 198,5 bis Elbe-km 230) }\end{array}$ & $\begin{array}{ll}\text { - } & \text { Geschiebezugabe } \\
\text { - } & \text { Geschiebeumlagerung } \\
\text { - } & \text { Modifikation der Rege- } \\
& \text { lungsbauwerke } \\
\text { - } & \text { Vergrößerung des Abfluss- } \\
& \text { anteils der Vorländer }\end{array}$ \\
\hline 5 & Coswig/Anh. & $\begin{array}{l}\text { Coswig/Anh. bis Mulde-Mündung } \\
\text { (Elbe-km } 230 \text { bis Elbe-km 259,6) }\end{array}$ & $\begin{array}{ll}\text { - } & \text { Geschiebezugabe } \\
\text { - } & \text { Geschiebeumlagerung } \\
\text { - } & \text { Modifikation der Rege- } \\
\text { lungsbauwerke } \\
\text { - Vergrößerung des Abfluss- } \\
\text { anteils der Vorländer }\end{array}$ \\
\hline 6 & Aken & $\begin{array}{l}\text { Mulde-Mündung bis Steckby } \\
\text { (Elbe-km 259,6 bis Elbe-km 280) }\end{array}$ & $\begin{array}{ll}\text { - } & \text { Geschiebeumlagerung } \\
\text { - } & \text { Modifikation der Rege- } \\
& \text { lungsbauwerke } \\
\text { - } & \text { Uferabgrabung } \\
\text { - } & \text { Vergrößerung des Abfluss- } \\
& \text { anteils der Vorländer }\end{array}$ \\
\hline 7 & Saalemündung & $\begin{array}{l}\text { Steckby bis Saalemündung } \\
\text { (Elbe-km } 280 \text { bis Elbe-km 290,7) }\end{array}$ & 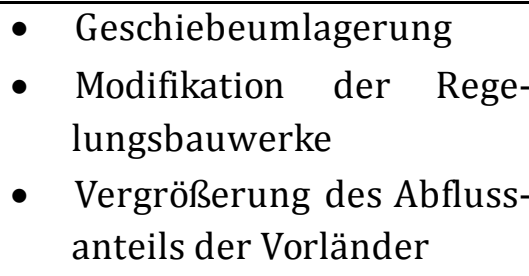 \\
\hline
\end{tabular}

Tab. 1: $\quad$ Streckenunterteilung der Erosionsstrecke mit jeweiligen Schwerpunktmaßnahmen 


\section{Autorenfassung}

Gabriel, Kühne, Faul haber, Promny, Horchler: Sohlenstabilisierung und Erosionseindämmung am Beispiel der Elbe, 2011

Gabriel, Kühne, Faulhaber, Promny, Horchler: Sohlenstabilisierung und Erosionseindämmung am Beispiel der Elbe. WasserWirtschaft 6 (2011), S. 27-32.

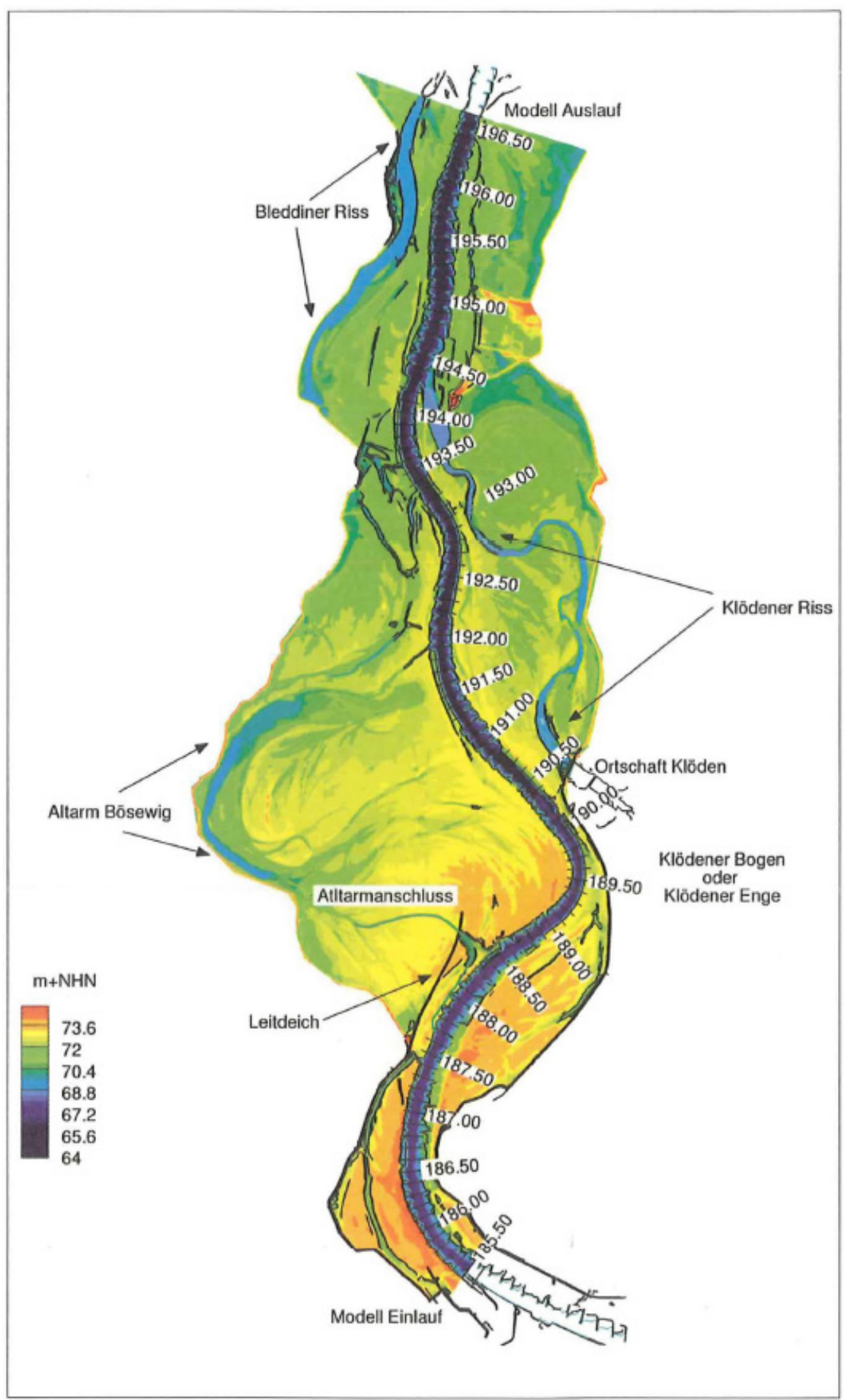

Bild 3: $\quad$ Übersichtskarte der Pilotstrecke Klöden mit einerVariante des Altarmanschlusses 


\section{Autorenfassung}

Gabriel, Kühne, Faul haber, Promny, Horchler: Sohlenstabilisierung und Erosionseindämmung am Beispiel der Elbe, 2011

sind, neben einer umfänglichen Erhebung von Naturdaten, auch hydraulisch-morphologische Modelluntersuchungen. Diese wurden schon im Rahmen der Konzeptentwicklung durch die BAW durchgeführt [2] und werden aktuell mit Hilfe eines neu aufgebauten mehrdimensionalen Strömungsmodells ergänzt. Mit dem Modell werden die Änderungen der Strömungsparameter 30 bei kleinräumiger Topografiemodifikation durch Baumaßnahmen untersucht. Die langfristigen hydraulisch-morphologischen Wirkungen der Maßnahmen werden mit hydronumerischen 1-DFeststofftransportmodellen für die gesamte Erosionsstrecke ermittelt [7]. Die Ergebnisse dieser Modelle werden für ökologische Untersuchungen der BfG (INFORM) (8] genutzt.

\section{Dreidimensionales numerisches Modell Klöden}

Die Untersuchungen zur Oberflächenströmung bei Annahme einer festen Sohle werden mittels eines dreidimensionalen hydronumerischen Strömungsmodells (3-D-HN-Modell) durchgeführt. Modellgrundlage ist dafür der Topografiezustand des Jahres 2003 für den Vorlandbereich und der des Jahres 2004 für die Stromsohle (Bild 3). Die horizontale Gitternetzauflösung beträgt 2 x $2 \mathrm{~m}$ im Gewässerbett und 5 x 5 m bzw. 10 x 10m auf dem Vorland, die vertikale Auflösung 0,5m [9].

Auf Basis der Analyse des bestehenden Zustands wurden verschiedene Varianten für den Bereich Klöden entworfen. Im Ergebnis bildeten sich zwei Gruppen von möglichen Maßnahmen heraus. Die Gruppe 1 hat Baumaßnahmen im Gewässerbett zum Untersuchungsgegenstand. Die Bauwerke werden um bis zu 0,7 m aufheutiges Mittelwasserniveau abgesenkt und der Streichlinienabstand vergrößert. Gleichzeitig wird die Engstelle am Klöd ener Bogen durch Vorlandabgrabung am Innenufer aufgeweitet und eine gleichmäßigere Stromführung erzeugt.Zu diesem Zwecke werden insbesondere die Übergänge von Strecken mit Buhnen zu Deckwerken gleichmäßiger gestaltet. Im oberstromigen Abschnitt des Modells werden auf der rechten Seite Buhnenfelder ausgeräumt, um die Reibungswirkung der Buhnenfeldwalzen zu reaktivieren.

Die Analyse des Ist-Zustandes hat ergeben, dass das Vorland für Abflüsse bis HQ2 vom Hauptgerinne abgeschnitten ist und dann durch Rückstau überflutet wird. Mit der Maßnahmengruppe 2 wird das Vorland auch für Abflüsse um doppeltes Mittelwasser (2MQ) wieder von Oberstrom mit dem Hauptgerinne vernetzt Dazu werden Altarme und Nebenrinnen reaktiviertbzw. alternative Rinnen geschaffen . Indem Durchflussanteile auch bei kleinem Hochwasser über das Vorland abgeleitet werden, kann die Fließgeschwindigkeit im Gewässerbett und damit die Belastung der Sohle verringert werden. Gleichzeitig helfen diese Maßnahmen, den Auencharakter des Vorlandes zu stärken. Die Untersuchung der Geländehöhen zeigt, dass aufgrund der hohen Vorländer Anbindungen nur an wenigen Stellen für Abflüsse $>2 \mathrm{MQ}$ denkbar sind. Eine Variante des Altarmanschlusses zeigt Bild 3.

Die Analyse der hydraulischen Veränderungen der Varianten gegenüber dem Ist-Zustand erlaubt eine Prognose darü- 

WasserWirtschaft 6 (2011), S. 27-32.

ber, inwieweit ein solches Paket baulicher Maßnahmen das Erosionsproblem entschärfen kann. Bild 4 zeigt einen Vergleich des heutigen Zustandes (Bild 4 oben) mit einer Studie, die die oben beschriebenen Maßnahmengruppen 1 und 2 miteinander verbindet (Bild 4 Mitte). Umgesetzt sind das Ausräumen der Buhnenfelder, die neue Streichlinie im Klödener Bogen sowie der Anschluss des Bösewiger Altarmes. In den nicht vom Wasser benetzten Arealen gilt die Grauwertcodierung über die Geländehöhe. In benetzten Arealen erfolgte die Farbzuweisung entsprechend der Fließgeschwindigkeiten. Mit wachsender Geschwindigkeit wird die Darstellung heller. Gut zu erkennen sind die durchströmten Bereiche im linken Vorland durch Anschluss des Altarmes und die Reduzierung der Fließgeschwindigkeiten in der Studie gegenüber dem Ist-Zustand auf der gesamten dargestellten Fließlänge im Flussschlauch (Bild 4 oben und Mitte). Der Vergleich der über die Tiefe, die Fahrrinnenbreite und auf 20m Fließlänge gemittelten Geschwindigkeiten (Bild 4 unten) verdeutlicht die Verringerung der Geschwindigkeiten und damit der Beanspruchung der Sohle durch das Maßnahmenpaket.

\section{Ökologische Wirkungsanalyse mit dem Modell INFORM}

Die Auswirkungen der Maßnahmen auf die Vegetation der Elbaue werden durch die BfG mit Hilfe des Modellsystems IN FORM [8] abgeschätzt. Basierend auf den Gegebenheiten im Ist-Zustand und den Modellergebnissen wird eine naturschutzfachliche Bewertung vorgenommen. Aufgrund einer oft engen Bindung der Vegetation der Flussauen an wenige Umweltfaktoren ist es möglich, bei Änderung dieser Faktoren modellhafteAbschätzungen der Veränderung der Pflanzenhabitate vorzunehmen. Da die wichtigsten Umweltfaktoren auch für die Maßnahmenvarianten als Rasterdaten vorliegen, können mit INFORM räumlich explizite Vorhersagen der potenziellen Änderung der Pflanzenhabitate getroffen werden. Durch die Verknüpfung der naturschutzfachlichen Bewertung mit den Rasterdaten kann der potenzielle Verlust und Gewinn an wertvollen Flächen bilanziert werden. Somit wird die Erstellung einer Rangfolge für die jeweiligen Maßnahmenvarianten aus naturschutzfachlicher Sichtmöglich. Eine entsprechende Reihungkann vorgenommen werden und mögliche Zielkonflikte werden dabei schon frühzeitig aufgezeigt.

\section{Umsetzung des Sohlenstabilisierungskonzeptes}

Die Eindämmungder Erosionstendenzen an der Elbe oberhalb der Saalemündung stellt eine umfangreiche Aufgabe für die WSV dar und wird aufgrund der Komplexitätder Problematikin direkter Abstimmung mit den betroffenen Bundesländern Sachsen und Sachsen-Anhalt durchgeführt. 


\section{Autorenfassung}

Gabriel, Kühne, Faul haber, Promny, Horchler: Sohlenstabilisierung und Erosionseindämmung am Beispiel der Elbe, 2011

Um die Umsetzung der in dem Sohlenstabilisierungskonzept vorgesehenen Maßnahmen in eine Flussgebietsbetrachtung einzubinden, in der die Belange der Schifffahrt, der Wasserwirtschaft, des Hochwasserschutzes sowie des Natur-, Kultur-und Umweltschutzes angemessen Berücksichtigung finden kön-

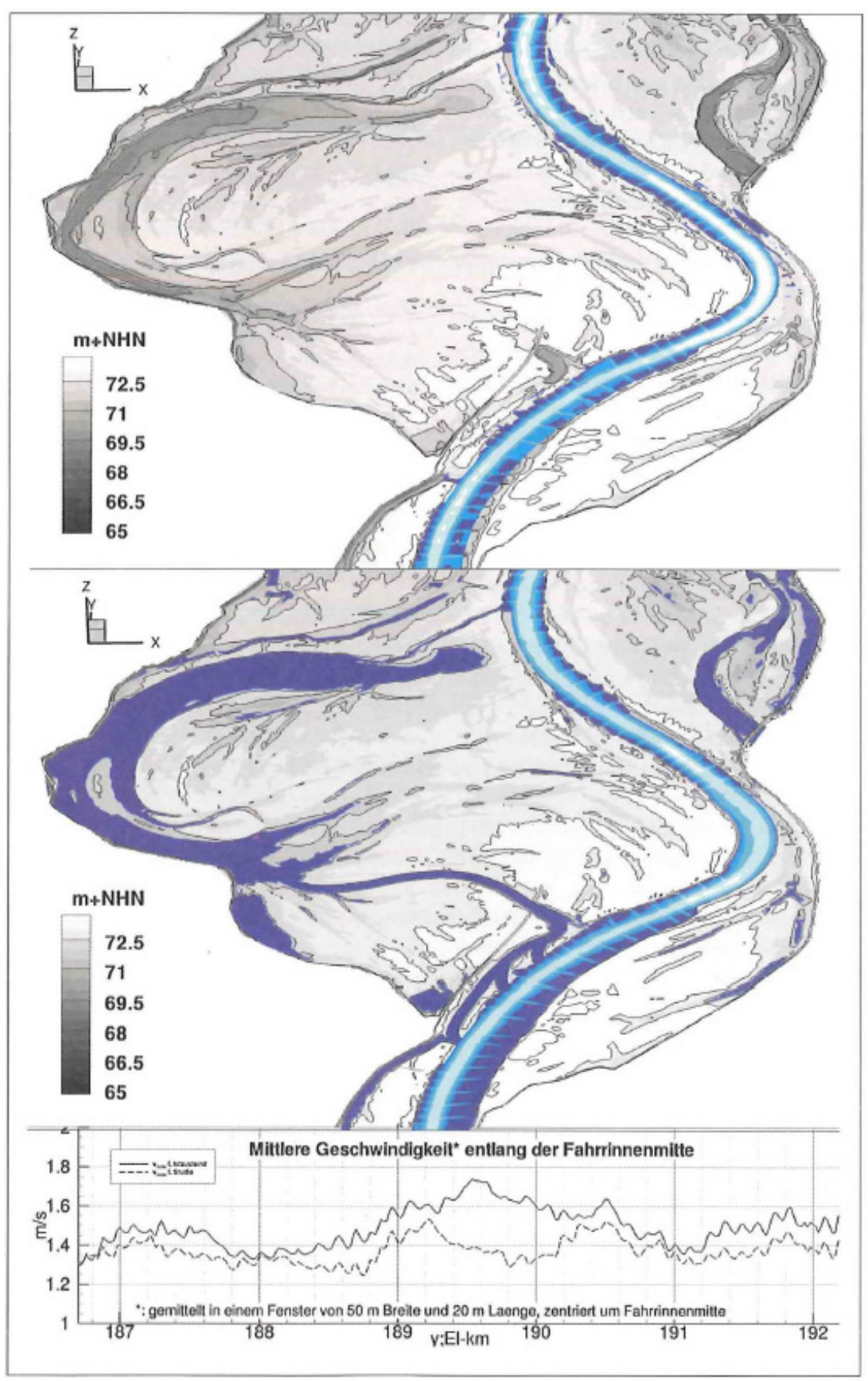

Bild 4: $\quad$ Über die Tiefe gemittelte Fließgeschwindigkeiten im Ist-Zustand (oben) und für eine Maßnahmestudie (Mitte) sowie Vergleich der für die Fahrrinne gemittelten Fließgeschwindigkeiten (unten; durchgezogene Linie: Istzustand, gestrichelt: Studie) (3-D-HNModell bei 2 MQ) 


\section{Autorenfassung}

Gabriel, Kühne, Faulhaber, Promny, Horchler: Sohl enstabilisierung und Erosionseindämmung am Beispiel der Elbe, 2011

\section{Gabriel, Kühne, Faulhaber, Promny, Horchler: Sohlenstabilisierung und Erosionseindämmung am Beispiel der Elbe. WasserWirtschaft 6 (2011), S. 27-32.}

nen, wurde ein Beirat eingerichtet. In dem Beirat sollen im Vorfeld der rechtlichen Verfahren und in Begleitung der Maßnahmen Informationen ausgetauscht werden.

Um neben Fachinstitutionen, Behörden und Umweltverbänden auch die interessierte Öffentlichkeit möglichst frühzeitig sowie umfänglich über geplante Maßnahmen vor Ort zu informieren und auch um über die Problematik der Erosion an der Elbe, ihre Ursachen und die jeweiligen Lösungsansätze zu unterrichten, hat die WSV ein Konzept zur Information der Öffentlichkeit entwickelt. Es wird damit angestrebt, das Sohlenstabilisierungskonzept als Konzept der WSV bekannt zu machen und eine größere Akzeptanz in der Öffentlichkeit bei der Durchführung baulicher Maßnahmen an der Elbe zu erzeugen.

Durch das WSA Dresden werden derzeit die planerischen und haushaltstechnischen Grundlagen für die Pilotmaßnahme Klöden erstellt. Es ist vorgesehen, noch in diesem Jahr mit dem Genehmigungsverfahren zu beginnen. Zeitlich versetzt sollen umfangreiche bauliche Maßnahmen in den anderen Streckenabschnitten der Erosionsstrecke in die konkrete Planung gehen. Unabhängig davon werden schon heute die Maßnahmen in der Erosionsstrecke der Elbe, die keiner planrechtlichen Genehmigung bedürfen, nach den Vorgaben und Kriterien des Sohlenstabilisierungskonzeptes durchgeführt. Damitsind für diesen Abschnitt der oberen Mitteleihe die Grundlagen für eine nachhaltige Erosionseindämmung auf den Weg gebracht.

Thomas Gabriel, Elke Kühne, Petra Faulhaber, Markus Promny and Peter Horchler

\section{Stream Bed Stabilisation and Erosion Mitigation exemplified by the Elbe River}

At a reach of the Middle Elbe river, the stream bed has lowered by an average of $1 \mathrm{~m}$ during the last more than 100 years. This erosive tendency is increasingly posing a threat to the functioning of the rivers' training structures. Furthermore, future environmental impacts cannot be ruled out. Based on a stream stability concept, the Federal Waterways and Shipping Administration is conducting necessary measures to minimize the erosion while maintaining the natural stream bed dynamics. 


\section{Autorenfassung}

Gabriel, Kühne, Faul haber, Promny, Horchler: Sohlenstabilisierung und Erosionseindämmung am Beispiel der Elbe, 2011

Томас Габриель, Эльке Кюне, Петра Фаульхабер, Маркус Промни и Петер Хорхлер

\section{Стабилизация русла и}

\section{сдерживание эрозии на примере реки Эльбы}

На участке Средней Эльбы русло реки углубилось в среднем на один метр в течение более чем 100-летнего периода. Данные эрозийные тенденции представляют собой все возрастающую угрозу для функционирования системы регулирования водотока. Не следует также исключать возможные воздействия в будущем на окружающую среду. На основании плана по стабилизации русла федеральным Управлением водными путями, водоснабжением и судоходством проводятсянеобходимыемероприятияполокализацииэрозиисодновременным сохранением естественной динамики русла.

\section{Autoren}

\section{Dipl.-Geogr. Thomas Gabriel}

Wasser- und Schifffahrtsdirektion Ost

Gerhart-Hauptmann-Straße 16

39108 Magdeburg

thomas.gabriel@wsv.bund.de

\section{Dipl.-Ing Elke Kühne}

Wasser- und Schifffahrtsamt Dresden Moritzburger Straße 1, 01127 Dresden elke.kühne@wsv.bund.de

\section{Dipl.-Ing. Petra Faulhaber}

Bundesanstalt für Wasserbau

Kußmaulstraße 17,76187 Karlsruhe

petra.faulhaber@baw.de

\section{Dr.-lng. Markus Promny}

Dr. rer. nat. Peter Horchler

Bundesanstalt für Gewässerkunde

Am Mainzer Tor 1, 56068 Koblenz

promny@bafg.de

horchler@bafg.de 


\section{Autorenfassung}

Gabriel, Kühne, Faul haber, Promny, Horchler: Sohlenstabilisierung und Erosionseindämmung am Beispiel der Elbe, 2011

\section{Literatur}

[1] Kühne, E.; Schoßig, R.: Geschiebezugabezur Sohlstabilisierung der Elbestrecke unterhalb Dresden. In: Wasserbaukolloquium des Instituts für Wasserbau und Technische Hydromechanik der TU Dresden (2000), S. 57-67.

[2] PG Erosionsstrecke Elbe (Hrsg.): Sohlstabilisierungskonzept für die Elbe von Mühlberg bis zur Saalemündung. 2009

(www.wsd-ost.wsv.de/betrieb_unterhaltung/Elbe/Erosion/index.html)

[3] Vollmer, S.; Schriever, S.: Feststoffhaushalt der Elbe. In: Veranstaltungen der Bundesanstalt für Gewässerkunde (2005), Nr. 4, S. 25-36.

[4] Faulhaber, P.: Maßnahmen gegen die Sohleintiefung der Elbe. ln : WasserwirtschaftWassertechnik (2001), Heft 7, S. 17-22.

(5] Anlauf, A.; Hentschel, B.: Untersuchungen zur Wirkung verschiedener Buhnenformen auf die Lebensräume in Buhnenfeldern der Elbe. In: Tagungsband des 10. Magdeburger Gewässerschutzseminars. Stuttgart: Teubner Verlag, 2002, S. 199-202.

[6] Faulhaber, P.: Entwicklung der Wasserspiegel und Sohlhöhen in der deutschen BinnenElbe innerhalb der letzten 100 Jahre- Einhundert Jahre "Elbestromwerk". In: Tagungsband des 8. Magdeburger Gewässerschutzseminar. Stuttgart: Teubner-Verlag, 1998, S. 217-220.

[7] Alexy, M: Feststofftransportmodell zur Simulation von Geschiebezugabeszenarien zur großräumigen und langfristigen Stabilisierung der Elbesohle in der Erosionsstrecke. In: Dresdner Wasserbauliche Mitteilungen (2004), Heft 27, S. 459-471.

[8] Fuchs, E.; Giebel, H.; Hettrich, A.; Hüsing, V.; Rosenzweig, S.; Theis H.-J.: Einsatzvon ökologischen Modellen in der Wasser- und Schifffahrtsverwaltung, Das integrierte Flussauenmodell INFORM (Version 2.0).1n: Mitteilungen der Bundesanstalt für Gewässerkunde (2003), Nr. 25.

[9] Patzwahl, R.; Jankowski, J.; Lege, T.: Very high resolution numerocal modelling for inland waterway design. In: Proceedings to River Flow 2008 (2008), Vol. 3, S. 2033-2041. 